'Observatorio de Comunicación y Salud. Universidad Austral de Chile-Universidad de la Frontera. Valdivia, Chile.

Instituto de Comunicación Social-Facultad de Filosofía y Humanidades, Universidad Austral de Chile. Valdivia, Chile. aAlumno Programa de Doctorado en Comunicación Universidad Austral de Chile- Universidad de la Frontera. Valdivia-Temuco, Chile.

${ }^{b}$ Periodista y Licenciada en Comunicación Social. M.A. in Communication Studies. Ingeniera en sonido. Magister en Desarrollo Humano. Master en Educación Experiencial. dPeriodista y Licenciado en Ciencias de la Comunicación. Magister en Percepción, Comunicación y Tiempos. Magister en Informática Educativa. Doctor en Percepción, Comunicación y Tiempos.

Trabajo no recibió financiamiento. Los autores declaran no tener conflictos de interés.

Recibido el 25 de agosto de 2020, aceptado el 23 de marzo de 2021.

Correspondencia a: Miguel Ángel Carrasco-García Beauchef \#765. Valdivia, Chile. carrascogarcia@gmail.com

\section{Representación de la incidencia y de la mortalidad por cáncer en los medios de comunicación chilenos}

\author{
MIGUEL ANGEL CARRASCO-GARCÍA ${ }^{1, \mathrm{a}}$, CAROLA NEIRA-MELLADO ${ }^{1,2, \mathrm{~b}}$, \\ BÁRBARA KLETT ${ }^{1, a, c}$, LUIS CÁRCAMO-ULLOA ${ }^{2, \mathrm{~d}}$
}

\section{Portrayal of cancer prevalence and mortality in Chilean digital media}

\begin{abstract}
Background: Cancer is the second death cause in Chile. The Chilean National Cancer Act will secure treatment and labor protection for people diagnosed with cancer. Aim: To answer questions regarding the media portrayal of cancer. Material and Methods: Through data-mining and the conduction of content analysis, 2,523 news titles about cancer were analysed. The news titles were obtained from 345 Chilean digital media which published cancer related news on Twitter between January and December 2019. An attempt was made to answer two research questions, namely are cancer incidence and mortality rates portrayed in a corresponding magnitude in the Chilean digital media? and what words are commonly used for this purpose? Results: There is not a coherence between the incidence and mortality of the main cancer types in Chile and the amount of content published in communication media. Conclusions: Our results are consistent with international studies. We should expect the delivery of complete, timely and effective information about cancer in communication media, aiming to educate the population and reinforce prevention.
\end{abstract}

(Rev Med Chile 2021; 149: 716-723)

Key words: Epidemiology; Health Communication; Medical Oncology; Public Health.
S egún la Organización Mundial de la Salud (OMS), el cáncer es la segunda causa de muerte en el mundo, siendo detectada mayoritariamente en una fase avanzada ${ }^{1}$. En 2017, más de $90 \%$ de los países de ingresos altos brindaron tratamiento a enfermos oncológicos, mientras que en países de ingresos bajos, este porcentaje fue inferior a 30\% para el mismo período ${ }^{1}$.

En las Américas, esta patología causó 1,3 millones de muertes en 2018 y se registraron 3,7 millones de nuevos casos, cifra que se calcula, llegará a 5 millones en $2023^{2}$. La Organización Panamericana de la Salud (OPS) estima que 40\% de los casos de cáncer podrían prevenirse evitando factores de riesgo como el tabaquismo, el consu- mo abusivo de alcohol, una dieta poco saludable y el sedentarismo. Además, en 30\% de los casos una detección temprana y tratamiento adecuado pueden curar la enfermedad ${ }^{2}$.

En Chile, en 2016, el cáncer fue la segunda causa de muerte, registrada solo después de padecimientos cardiovasculares, y para 2023 se espera que sea la principal ${ }^{3}$. Los cánceres con mayor mortalidad e incidencia en Chile en hombres son el de estómago, próstata y pulmón, mientras que en mujeres son mama, pulmón y vesícula y vías biliares ${ }^{4}$ (Tabla 1 ).

En relación al cáncer pediátrico, al año se diagnostican en rango entre 490 y 500 niños, correspondiendo a $1 \%$ del total de los cánceres diagnosticados en el país ${ }^{5,6}$ (Tabla 2). 
Tabla 1. Estimaciones de incidencia de cáncer en mujeres y hombres, según orden de magnitud. Chile 2003-2007

\begin{tabular}{|c|c|c|c|}
\hline $\begin{array}{l}\text { Mujeres } \\
\text { Localización del tumor }\end{array}$ & Incidencia* & $\begin{array}{l}\text { Hombres } \\
\text { Localización del tumor }\end{array}$ & Incidencia* \\
\hline Mama & 46,2 & Próstata & 50,9 \\
\hline Piel no melanoma & 22,8 & Estómago & 29,7 \\
\hline Vesícula biliar y vías biliares & 18,7 & Piel no melanoma & 19,6 \\
\hline Cuello uterino & 15,6 & Tráquea, bronquios y pulmón & 17,1 \\
\hline Estómago & 14,3 & Colon & 9,3 \\
\hline Colon & 10,7 & Testículo & 8,0 \\
\hline Tráquea & 9,9 & Vesícula biliar & 7,5 \\
\hline Ovario & 6,7 & Esófago & 6,9 \\
\hline Linfoma no Hodgkin & 6,4 & Riñones & 6,8 \\
\hline Tiroides & 5,9 & Leucemia & 6,1 \\
\hline
\end{tabular}

*Tasa bruta de incidencia por 100.000 habitantes. Fuente: (3).

Tabla 2. Mortalidad por tumores malignos en mujeres y hombres. Chile 2015

\begin{tabular}{|lclc|}
\hline Mujeres & Hombres & Localización del tumor & \multicolumn{1}{c|}{ Mortalidad* } \\
\hline Mama & 16,6 & Estómago & 25,5 \\
\hline Tráquea, bronquios y pulmón & 13,8 & Próstata & 23,5 \\
\hline Vesícula biliar y vías biliares & 12,5 & Tráquea, bronquios y pulmón & 20,7 \\
\hline Estómago & 12,3 & Colon & 9,7 \\
\hline Colon & 11,0 & Hígado & 7,7 \\
\hline Páncreas & 7,8 & Páncreas & 7,2 \\
\hline Cérvico Uterino & 6,8 & Vesícula biliar & 5,9 \\
\hline Hígado & 6,4 & Riñones & 5,7 \\
\hline Ovario & 4,8 & Esófago & 4,3 \\
Leucemia & 4,2 & Vejiga & 4,2 \\
\hline
\end{tabular}

*Tasa cruda por 100.000 habitantes. Fuente: (3).

\section{Principales factores de riesgo}

La edad es un factor de riesgo en la adquisición del cáncer tanto en hombres como en mujeres. La mortalidad por cáncer de mama en mujeres chilenas se incrementa conforme aumenta la edad, presentando una fuerte alza a partir de los 80 años ${ }^{7}$. En el caso del cáncer de próstata, en tanto, el principal factor de riesgo es la edad y en menor medida, los antecedentes familiares ${ }^{8}$.
En el caso del cáncer de pulmón, el tabaquismo es el factor asociado más determinante, aumentando el riesgo de contraer la enfermedad entre 15 a 30 veces. Más aun, en Chile se ha estimado que $85 \%$ de los cánceres de pulmón en hombres y $40 \%$ en mujeres son explicados por consumo de tabaco 9 . En cuanto al cáncer de vesícula biliar, el principal factor de riesgo es la presencia de cálculos biliares ${ }^{10,11}$. Respecto al cáncer gástrico, la infección por H. pylori parece ser el factor más importante 
¿La prensa puede distorsionar la presencia del cáncer? - M. A. Carrasco-García et al

en $60-70 \%$ de los pacientes con cáncer no cardial; mientras que la obesidad y el reflujo gastroesofágico parecieran tener un rol en la patogenia del cáncer cardial ${ }^{12}$.

El cáncer en Chile tiene un impacto económico de más de 2.100 millones de dólares al año, lo que representa casi $1 \%$ del producto interno bruto del país. El gasto directo per cápita fue de 47 dólares $^{13}$.

\section{Los medios de comunicación y la construcción de la realidad}

Los medios de comunicación son capaces de influir en la construcción de la percepción de la sociedad e influir en la instalación de la perspectiva moral y solidaria ${ }^{14}$. El rol de los medios comienza desde el momento en que la prensa selecciona aconteceres para publicarlos, imprimiendo una visión y abriendo la posibilidad de manipular los datos de referencia ${ }^{15}$.

Esa representación social de la realidad intenta condensar cómo piensan sus integrantes, desde una lógica social, arraigada en el saber común y orientada a la comunicación, entendimiento y dominio del entorno ${ }^{16}$.

Saber qué escriben los medios de comunicación sobre el cáncer ha sido objeto de diversas investigaciones, las que han generado un extenso cuerpo de conocimiento en el área de medios

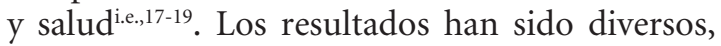
dando cuenta de coberturas visibilizadoras de factores de riesgo asociados al cáncer, conflictos con prestadores de salud y nuevos tratamientos ${ }^{17}$.

Igualmente, se ha indagado el uso de la metáfora en la presentación de terapias contra el cáncer definiendo conceptos predominantes como "armas", "ropa", "comida" y "cócteles"18. La cobertura de nuevas vacunas contra el cáncer también ha sido explorada, concluyendo que el mensaje predominante corresponde a "efectos adversos"19.

Las redes sociales también son un espacio para hablar de cáncer. Plataformas como Facebook han sido exploradas en distintos países (Argentina, Chile, Colombia y España), evidenciando una mayor actividad de difusión de esta enfermedad durante períodos de campañas preventivas, pero apuntando a un tratamiento genérico ${ }^{20}$.

Los análisis de contenidos in extenso han dado cuenta de coberturas superficiales ${ }^{21}$ y desequilibradas en relación al perfil epidemiológico del cáncer $^{22}$. En efecto, los resultados demuestran que la edad, como factor, y los adultos mayores, como grupo, son invisibilizados en la representación mediática del cáncer y, peor aun, los factores de riesgo asociados no son abordados en profundidad ${ }^{21}$. En medios de prensa japoneses, los cánceres gástricos y de colon son los menos abordados, a pesar de tener la mayor mortalidad ${ }^{22}$. En la República de Corea, en tanto, se determinó que existe una cobertura distinta del cáncer cuando hay factores publicitarios involucrados o cuando la agencia era conducida gubernamentalmente, generando un impacto negativo en las brechas de conocimiento $y$ en las inequidades en salud ${ }^{23}$.

Exhibiendo la influencia de los medios de comunicación como fuente de información y educación, es pertinente analizar el tratamiento de temas de alta sensibilidad en salud pública, como es el cáncer y conocer cómo se proyecta a la comunidad. Lo anterior, considerando que las patologías oncológicas representan la segunda causa de muerte en Chile y asumiendo que la información difundida en los medios podría sensibilizar y motivar la detección temprana y adquisición de conductas preventivas en la población. Este trabajo tiene como objetivo cuantificar la cobertura en los medios de prensa escrita digital de Chile. Para ello, las preguntas de investigación que guían este trabajo son: ¿Las tasas de incidencia y mortalidad por cáncer en Chile se reflejan en la cobertura de los medios de prensa escrita digital en su correspondiente magnitud? Y cuando se habla de cáncer ¿cuál es su cotexto? (¿qué palabras, adjetivos o términos acompañan al cáncer en una lógica descriptiva?).

\section{Metodología}

Para la presente investigación, se empleó un crawler informático ad-hoc para la exploración de las menciones en la prensa chilena ${ }^{24,25}$. Esta herramienta permite explorar, ordenar y construir casos noticiosos a partir de los datos textuales que publican los medios informativos. El crawler almacena, cada ocho minutos, todas las noticias que 350 medios chilenos vierten en la red social Twitter, registrando sus titulares y permitiendo acceder a cada noticia en extenso. De este modo, la presente investigación se define como un análisis de contenido exploratorio a partir de minería de textos periodísticos. 


\section{Búsqueda de datos}

La estrategia de búsqueda de datos produjo un total de 3725 textos para las menciones $<$ cáncer $>$ $+<$ cancer $>+<$ oncológico $>+<$ oncologico $>+$ $<$ oncología $>+<$ oncologia $><$ leucemia $>+$ $<$ linfoma $>+<$ neoplasia $>+<$ tumor $>$ tanto en el texto de tweet que publica el medio que generalmente es equivalente al titular de la noticia en la web como en las URL publicadas entre enero y diciembre de 2019. Posteriormente, los datos fueron filtrados manualmente para eliminar entradas duplicadas, resultando 2.435 textos.

Para la realización del análisis de contenido de los titulares, se procedió a elaborar un diccionario de etiquetas basales del cáncer, construyéndolas a partir de sinónimos y términos técnicos recurrentes para referirse a cada tipo de cáncer registrado en el perfil epidemiológico elaborado por el Ministerio de Salud ${ }^{3}$.

\section{Análisis de contenido}

Del total de titulares, se identificaron y cuantificaron las etiquetas basales de los distintos cánceres. Como resultado, se extrajo la frecuencia de las etiquetas para cada titular. Además, para abordar el cotexto del cáncer, fueron considerados los cuatro tipos con la mayor tasa de incidencia y los dos con la mayor frecuencia de mención. Para este análisis, se exploró la coocurrencia de palabras en el título de cada noticia en relación a la etiqueta basal de cáncer identificada en cada titular. Para ello, se utilizó una ventana de diez palabras (antecesoras y sucesoras) desde la posición de la etiqueta en el titular. Esta relación se presentó en una "nube de palabras", definida como una representación gráfica de la frecuencia de términos que acompañan a una palabra determinada con la finalidad de dar cuenta de la composición o idea general del texto evaluado ${ }^{26}$. La nube de palabras se formó a partir de los 50 términos más frecuentes de la coocurrencia. Todos estos análisis fueron realizados con los paquetes readtext ${ }^{27}$ y quanted $a^{28}$ en la plataforma estadística R v.3.6.329.

\section{Resultados}

Los resultados de las frecuencias de mención de los cánceres demuestran, en términos generales, que existe un reconocimiento y divulgación de noticias relacionadas a las diferentes variantes de la enfermedad. La neoplasia más citada en titula- res de medios chilenos fue la leucemia, seguido del melanoma, próstata, pulmón y mama. En el otro extremo, los menos nombrados fueron el cáncer testicular y de esófago con cero menciones (Figura 1).

En términos generales, los cánceres con mayor incidencia y mortalidad, tanto en hombres como en mujeres, no reciben la mayor cantidad de menciones. Cuando esta cobertura se compara con las tasas de incidencia en hombres ${ }^{3}$, los cánceres de próstata, estómago, piel no melanoma, tráquea-bronquios-pulmón y colon son los más preponderantes; sin embargo, de acuerdo a su frecuencia de mención, próstata, pulmón y colon aparecen citados en tercer, cuarto y sexto lugar en los medios, respectivamente, mientras que el cáncer de estómago aparece en el octavo puesto. La incidencia en mujeres, en tanto, está liderada por el cáncer de mama, seguido por piel no melanoma, vesícula y vías biliares, cuello uterino y estómago. De acuerdo a su frecuencia de mención, mama ocupa el quinto lugar, piel no melanoma no aparece citado, vesícula biliar se posiciona en el lugar decimoséptimo en frecuencia de menciones, mientras que el cáncer cérvicouterino en el décimo.

Cuando se contraponen frecuencia de mención y mortalidad ${ }^{3}$ en hombres, los tres cánceres con mayores tasas de mortalidad, estómago, próstata y cáncer de tráquea, bronquios y pulmón, se registran en octavo, tercer y cuarto lugar, respectivamente. En el caso de mortalidad del cáncer en mujeres, los tres cánceres con mayor tasa, mamas, bronquios y pulmón, y vesícula, se registraron quinto, cuarto y decimoctavo, de acuerdo a su frecuencia de mención.

La leucemia corresponde a un caso extremo, ya que si bien es un padecimiento con bajas tasas de incidencia y mortalidad, concentra la mayor frecuencia de mención en los medios. Un caso similar es observado para el melanoma de piel, con una tasa de mortalidad de las menores registradas ${ }^{3}$, pero con la segunda mayor frecuencia de mención.

Cabe mencionar el caso del cáncer testicular y de esófago, cuya tasa de incidencia y mortalidad es similar a la de otros cánceres en hombres, sin embargo, no concentran menciones en los titulares observados.

En cuanto al cotexto, se puede decir que los cánceres de próstata y mama comparten un 


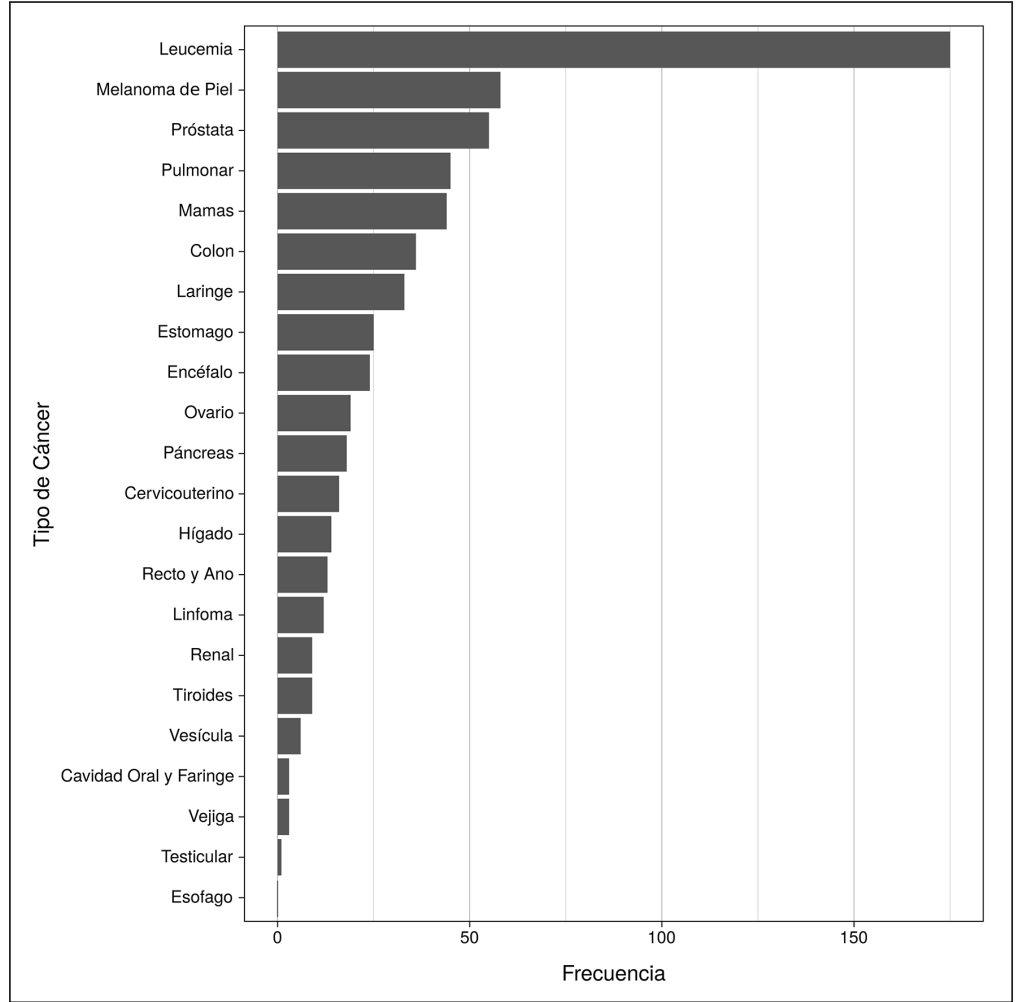

Figura 1. Frecuencia de menciones de los diferentes tipos de cáncer en titulares de medios digitales. Fuente: elaboración propia. dominio manejado desde la prevención, detección temprana y sensibilización, manifestado en términos como exámenes, campaña, operativo y temprana (Figura 2 a-b). En menor medida, se distingue una dimensión del costo asociado a la enfermedad, expuesto en palabras como costo y gratuito. El cáncer de estómago, en tanto, presenta un cotexto basado en la visibilización a través de (a)

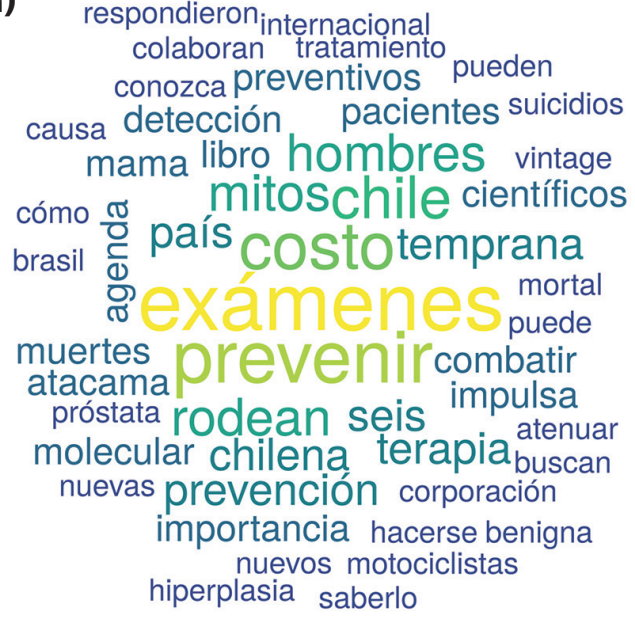

(b)

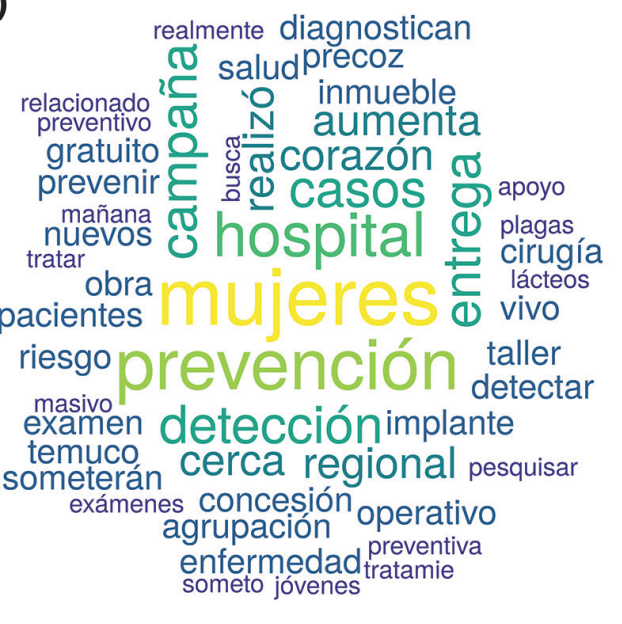

Figura 2. Nube de palabras cáncer de próstata (a) y cáncer de mama (b). 


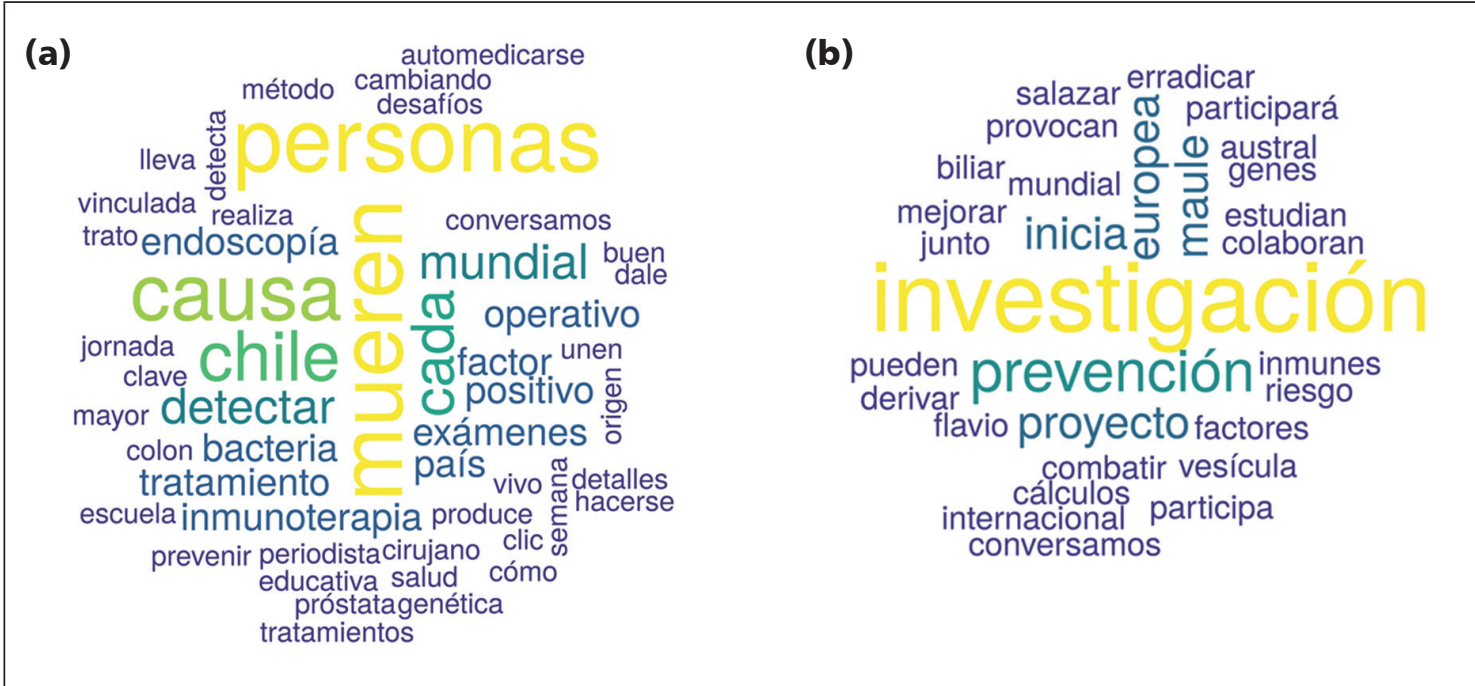

Figura 3. Nube de palabras cáncer de estómago (a) y cáncer de vesícula biliar (b).

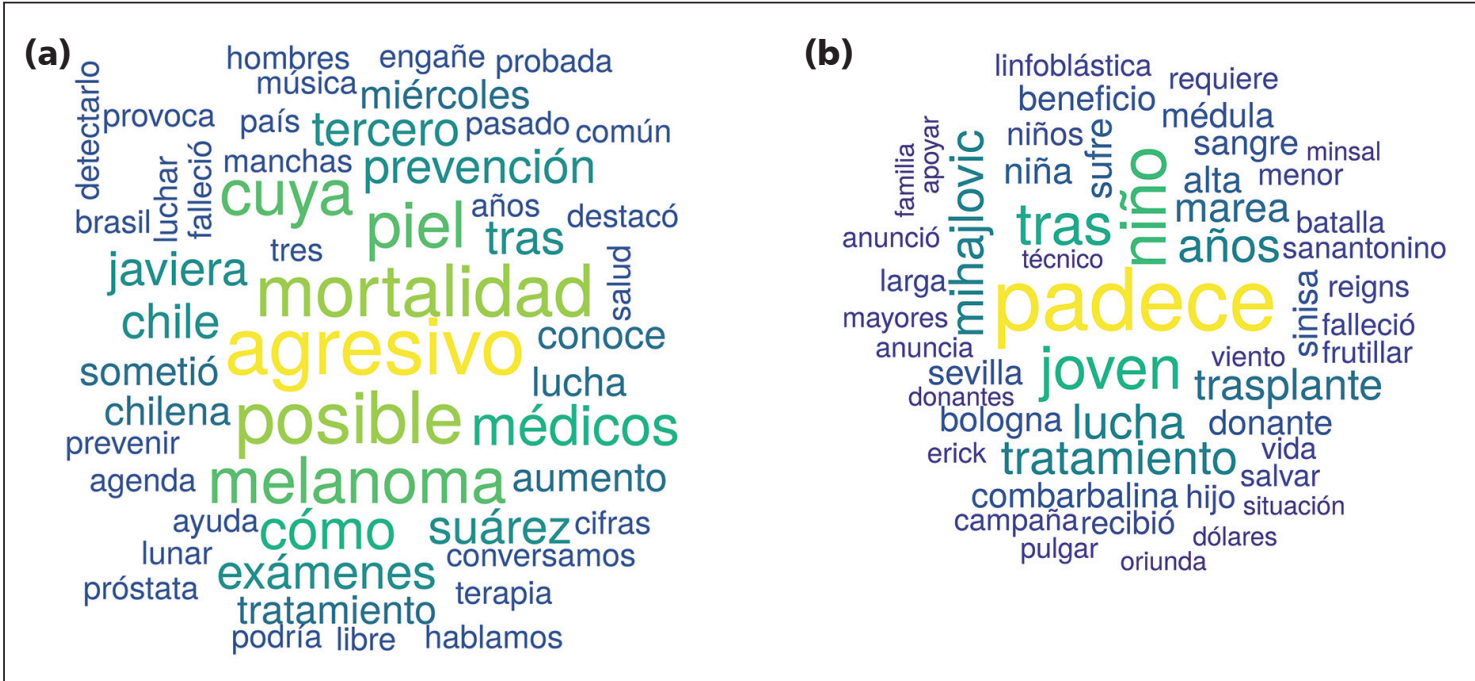

Figura 2. Melanoma de piel (a) y leucemia (b).

las consecuencias. Esto queda de manifiesto con palabras como personas, mueren y causa (Figura 3a). Para el caso del cáncer de vesícula biliar, el término dominante es investigación, lo que da cuenta de un dominio científico, técnico (Figura $3 b$ ). En estos dos últimos tipos, no se registran palabras que reflejen el sexo de quienes padecen la enfermedad. Finalmente, tanto el melanoma de piel como leucemia están descritos en un cotexto del miedo, manifiesto en palabras como mortalidad, agresivo y padece (Figura $4 \mathrm{a}-\mathrm{b}$ ). En menor medida, otros términos como batalla, lucha, niños y Javiera, dan cuenta de quienes padecen la enfermedad y desde donde se le aborda. 


\section{Discusión}

Los cánceres que reciben más cobertura son aquellos que afectan a personajes públicos o tienen un perfil asociado a lo emotivo como es el caso de la leucemia y el melanoma de piel, los cuales, a pesar de su baja incidencia, fueron los más citados en la prensa durante 2019. La alta mención de la leucemia se debe a que afecta principalmente a niños y los medios dan cuenta de esta condición (Figura 4b) enfocando el relato en el largo aliento de la enfermedad y en la sobrevida como lucha. El melanoma de piel, en tanto, también responde a la lógica hiperemotiva, esta vez, representado en el caso de la periodista Javiera Suárez. En resumen, ambas neoplasias operan bajo pulsos de información, respondiendo al ciclo de la noticia, pero siempre desde la lógica de lo emotivo y sin mayor profundización en aspectos clínicos, conforme a otros resultados ${ }^{20,22}$.

En una vereda opuesta se sitúan los cánceres de próstata y mama, los que están subrepresentados si se consideran sus tasas de incidencia y mortalidad. Sin embargo, cuando se analiza el contexto, se observa que están abordados desde las campañas preventivas, operativos y detección temprana, lo que habla de enfermedades con un asentamiento en los medios.

Existen también aquellos cánceres con una baja cobertura en medios, pero alta tasa de incidencia y mortalidad, como son el de estómago y el de vesícula biliar, cuya descripción es elaborada desde una lógica de lo técnico. Se habla de investigación, estudios asociados, tratamientos y detección, lo que implica un enfoque de tipo educativo e informativo para abordar la enfermedad.

Finalmente, cabe destacar que esta distorsión entre el volumen de cobertura de noticias sobre cáncer e incidencia de las patologías neoplásicas es consistente con otras investigaciones, siendo superficial ${ }^{21}$ y desequilibrada según el perfil epidemiológico del cáncer ${ }^{22}$, invisibilizando los factores de riesgo como el sexo y la edad, lo que impide desarrollar conductas preventivas en la población y un diagnóstico temprano.

Así también, se debe indagar el impacto que podrá tener la promulgación de la nueva ley en la representación que los medios realizan sobre el cáncer (Ley N 21.258/ 2 de septiembre 2020) desde el enfoque de comunicación y salud. Lo anterior para evitar distorsiones en la cobertura y potenciar el rol de informantes de los medios de prensa.

La presente investigación debe reportar limitaciones en lo conciso de las unidades de análisis seleccionadas (titulares). Investigaciones que extiendan la observación hacia el cuerpo de la noticia podrían entregar información que complementará la arrojada por el presente estudio.

\section{Referencias}

1. Organización Mundial de la Salud [Internet]. Notas Descriptivas: Cáncer; c2020 [citado el 15 de agosto de 2020]. Disponible en https://www.who.int/es/newsroom/fact-sheets/detail/cancer

2. Organización Panamericana de la Salud [Internet]. Enfermedades no Transmisibles y Salud Mental: Cáncer; c2020 [citado el 15 de agosto de 2020]. Disponible en https://www.paho.org/hq/index.php?option=com_content\&view $=$ article\&id=292: cancer-program \&Itemi$\mathrm{d}=3904$ \&lang=es

3. Ministerio de Salud-Gobierno de Chile [Internet]. Plan Nacional del Cáncer 2018-2028; c2020 [citado el 14 de agosto de 2020]. Disponible en https://www.minsal.cl/ wp-content/uploads/2019/01/2019.01.23_PLAN-NACIONAL-DE-CANCER_web.pdf

4. Mardones ML, Frenz P. Mortalidad por cáncer de vesícula y egresos hospitalarios por patología biliar en Chile 2002-2014, en relación a la garantía GES colecistectomía preventiva. Rev Med Chile 2019; 147 (7): 860-9.

5. PINDA Chile [Internet]. Estadísticas; c2020 [citado el 15 de agosto de 2020]. Disponible en https://www. pindachile.cl/estadisticas/

6. Ministerio de Salud-Gobierno de Chile [Internet]. Primer Informe del Registro Nacional de Cáncer Infantil de Chile (menores de 15 años), RENCI; c2018 [citado el 15 de agosto de 2020]. Disponible en https://www. pindachile.cl/estadisticas/

7. Icaza G, Núñez L, Bugueño H. Descripción epidemiológica de la mortalidad por cáncer de mama en mujeres en Chile. Rev Med Chile 2019; 145 (1): 106-14.

8. Ministerio de Salud-Gobierno de Chile [Internet]. Guia clinica próstata en personas de 15 años y más 2010; c2010 [citado el 14 de agosto de 2020]. Disponible en https://www.minsal.cl/portal/url/item/722233c6b946cd08e04001011f011d5e.pdf

9. Erazo M, Burotto M, Gormaz JG. Cáncer pulmonar, el peor efecto del tabaquismo. Rev Chil Enferm Respir. 2017; 33 (3): 219-21.

10. Roa JC, Ferreccio C, Miquel JF. Cáncer de la vesícula 
biliar: estudios necesarios para el diseño de estrategias de prevención y diagnóstico precoz. Rev Méd Clin Condes 2011; 22 (4): 486-91.

11. Latorre G, Ivanovic-Zuvic D, Corsi Ó, Valdivia G, Margozzini P, Olea R, et al. Cobertura de la estrategia preventiva de cáncer de vesícula biliar en Chile: Resultados de la Encuesta Nacional de Salud 2009-2010. Rev Med Chile 2015; 143 (2): 158-67.

12. Csendes A, Figueroa M. Situación del cáncer gástrico en el mundo y en Chile. Rev Chil Cir. 2017; 69 (6): 502-7.

13. Cid C, Herrera C, Rodríguez R, Bastías G, Jiménez J. Impacto económico del cáncer en Chile: una medición de costo directo e indirecto en base a registros 2009. Medwave 2016; 16 (7): e6509.

14. Luhmann N. La realidad de los medios de masas. Rubi: Universidad Iberoamericana; 2000.

15. Serrano M. Teoría de la Comunicación. Madrid, España: Editorial McGraw Hill; 2007.

16. Jodelet D. La representación social: fenómenos, concepto y teoría. En: Moscovici S, editor. Psicología Social II: Pensamiento y Vida Social. Barcelona: Paidós 1986; 469-94.

17. García A, Peris M, De Semir V, Borràs JM, Revuelta G, Méndez E, et al. Las noticias sobre el cáncer en los medios de comunicación escrita. Gaceta Sanitaria 2000; 14 (2): 139-45.

18. Camus JTW. ¡Acierta con la metáfora! Las metáforas de los tratamientos contra el cáncer en la prensa inglesa y española. Alfinge. Revista de Filología 2016; 109-38.

19. Tuells J, Duro JL, Chilet E, Pastor E, Portero A, Navarro C, et al. Noticias sobre el virus del papiloma humano y su vacuna en la prensa valenciana (2006-2011). Gaceta Sanitaria 2013; 27 (4): 374-7. https://dx.doi. org/10.1016/j.gaceta.2012.12.014

20. Fernández-Gómez E, Díaz-Campo J. Comunicación sobre el cáncer en Facebook: Las asociaciones de Argentina, Chile, Colombia y España. Cuadernos. Info 2016; (38): 35-50.

21. Macdonald S, Cunningham Y, Patterson C, et al. Medios de comunicación y factores de riesgo de cáncer: la infrarrepresentación de la edad. BMC Public Health 2018; 18: 490.

22. Miyawaki R, Shibata A, Ishii K, Koichiro O. Cobertura de noticias sobre el cáncer en periódicos japoneses: análisis de contenido. Comunicación en salud 2017; 32 (4): 420-6.

23. Min HS, Yun EH, Park J, Kim YA. [Cobertura de noticias sobre el cáncer en los periódicos coreanos: un estudio analítico en términos de concientización sobre el cáncer]. J Prev Med Public Health 2020; 53 (2): 126-34. https://doi.org/10.3961/jpmph.19.256

24. Cárcamo-Ulloa L, Calva-Rosales D, Ronquillo-Rodríguez N, Nesbet-Montecinos F. México, en la prensa chilena: análisis basado en minería de datos textuales en Twitter. Revista Latina de Comunicación Social 2017; 72: 897-914. http://www.revistalatinacs.org/072paper/1199/RLCS-paper1199.pdf.

25. Cárcamo-Ulloa L, Vernier M, Scheihing E, Aravena M, Pérez J. Sophia: una herramienta para la construcción y análisis de casos noticiosos en la enseñanza del periodismo. Nuevas Ideas en Informática Educativa 2017; 13: 667-72. https://www.academia.edu/35917012/Sophia una_herramienta_para_la_construccio_n_y_ana_lisis_ de_casos_noticiosos_en_la_ensenanza_del_periodismo

26. McNaught C, Liam P. Using wordle as a supplementary research tool. The Qualitative Report 2010; 15 (3): 63043.

27. Benoit K, Obeng A. Readtext: Import and handling for plain and formatted text files. $\mathrm{R}$ package version 0.76. [software]. 2020 [citado el 14 de agosto de 2020]. Disponible en https://CRAN.R-project.org/package $=$ readtext

28. Benoit K, Watanabe K, Wang H, Nulty P, Obeng A, Müller S, et al. Quanteda: an R package for the quantitative analysis of textual data. J Open Source Software 2018; 3 (30): 774 .

29. R Core Team. R: a language and environment for statistical computing. R foundation for statistical computing [software]. 2020. Vienna, Austria. 